\title{
Article \\ Electroosmosis and Electric Conduction of Electrolyte Solutions in Charge-Regulating Fibrous Media
}

\author{
Wei Lun Chen (1) and Huan Jang Keh *(1) \\ Department of Chemical Engineering, National Taiwan University, Taipei 10617, Taiwan; r05524102@ntu.edu.tw \\ * Correspondence: huan@ntu.edu.tw; Tel.: +886-2-33663048
}

check for updates

Citation: Chen, W.L.; Keh, H.J.

Electroosmosis and Electric

Conduction of Electrolyte Solutions

in Charge-Regulating Fibrous Media.

Colloids Interfaces 2021, 5, 19.

https: / / doi.org/10.3390/

colloids5010019

Academic Editors:

Emiliy Zholkovskiy and

Reinhard Miller

Received: 15 February 2021

Accepted: 15 March 2021

Published: 19 March 2021

Publisher's Note: MDPI stays neutral with regard to jurisdictional claims in published maps and institutional affiliations.

Copyright: (c) 2021 by the authors. Licensee MDPI, Basel, Switzerland. This article is an open access article distributed under the terms and conditions of the Creative Commons Attribution (CC BY) license (https:/ / creativecommons.org/licenses/by/ $4.0 /)$.

\begin{abstract}
An analytical study of the electroosmosis and electric conduction of electrolyte solutions in a fibrous medium composed of parallel charge-regulating cylinders with arbitrary electric double layer thickness is presented. A linearized charge regulation model was adopted for the association and dissociation reactions occurring at the amphoteric functional groups over the surfaces of the cylinders, and a unit cell model was employed to accommodate interactions among the cylinders. The electrokinetic equations governing the ionic concentration, electric potential, and liquid flow fields were solved at low zeta potential for the cylinders. Explicit formulas for the electroosmotic mobility and effective electric conductivity in the fiber matrix were obtained. The results indicate that the charge regulation characteristics, such as the equilibrium constants of the reactions occurring at the cylinders' surfaces and the bulk concentration of the charge-determining ions, influence the surface charge density and potential, electroosmotic mobility, and effective electric conductivity substantially.
\end{abstract}

Keywords: electroosmotic velocity; electrophoretic velocity; effective electric conductivity; charged cylinder; charge regulation; arbitrary electric double layer

\section{Introduction}

When a charged narrow channel or porous medium filled with an electrolyte solution is subject to an external electric field, an electroosmotic flow of the fluid occurs. Electroosmotic flows are of fundamental and practical interest in a variety of scientific and technological areas. The basic formulas of electroosmosis have been derived mainly for single microchannels or nanochannels [1-5].

In practical applications of electroosmosis, porous media are usually encountered. To avoid the difficulty of multiple-entity boundary value problems, the unit cell model was frequently used to predict the effects of interactions among the small solid entities constructing the porous medium on the electroosmotic velocity and effective electric conductivity. Although different shapes of the cell may be employed, a spherical [6-14] or circular cylindrical [15-20] one for the fictitious envelope of fluid surrounding each spherical or circular cylindrical entity is most convenient.

The previous studies of the electroosmotic velocity and electric conductivity in porous media, it was often assumed that the charge density or electric potential at the solid surface is constant. However, the charge density and electric potential of most biological and some inorganic surfaces are governed by some association and dissociation reactions, and the number density of their ionogenic functional groups. The extent of these reactions depends on the local concentration of the charge-determining or potential-determining ions, and other relevant parameters [21-27]. When such a charge-regulating surface is subject to an external electric field, the electric potential and ionic concentration distributions around will be influenced by the charge regulation characteristics and the fluid adjacent to the surface undergoes electroosmosis accordingly.

The electroosmosis and electric conduction of electrolyte solutions in long capillary tubes or slits whose inside walls were covered by a layer of adsorbed charge-regulating 
polymer of arbitrary thickness were analytically studied for the case of an arbitrary electric double layer, and closed-form formulas for the local and average electroosmotic velocities and electric current density were obtained for a linearized charge regulation model [28]. Recently, the electroosmotic mobility and ionic conductance in a long charge-regulating nanochannel of arbitrary cross-section but low surface potential have been semi-analytically examined, and the geometrical configuration of the nanochannel was shown to play a key role in the determination of these transport properties [29]. On the other hand, the electroosmosis/electrophoresis and electric conduction in a suspension or porous system of an assemblage of charge-regulating spherical entities have been investigated using the cell model, and explicit formulas for the electroosmotic/electrophoretic mobility and effective electric conductivity were derived as functions of the porosity and other relevant parameters [30].

In this paper, the unit cell model is used to analyze the electroosmotic mobility and effective electric conductivity in a fiber matrix constructed by a uniform array of parallel charge-regulating circular cylinders surrounded by arbitrary double layers. The closedform results enable these transport properties to be easily predicted for various values of the charge regulation characteristics and porosity of the fibrous system.

\section{Analysis}

Consider an arbitrary fluid solution having $M$ ionic species filled in a fiber matrix constructed by a homogeneous array of parallel, circular, dielectric cylinders. Each cylinder is long and has a charge-regulating surface whose ionogenic functional groups are in chemical equilibrium with the ambient solution. When the fiber matrix is subject to a transversely imposed electric field $E_{\infty} \mathbf{e}_{x}$, the fluid undergoes electroosmosis with a superficial velocity $-U \mathbf{e}_{x}$, where $\mathbf{e}_{x}$ is the unit vector in the $x$ direction. As illustrated in Figure 1, we use a unit cell model in which each cylinder with radius $a$ is enveloped by a coaxial annulus of the fluid with outer radius $b$, and the fluid-to-cell volume ratio equals the porosity $1-\varphi$ of the fibrous system; i.e., $\varphi=(a / b)^{2}$. The origin of the polar coordinate system $(\rho, \phi)$ is set at the cylinder axis with $x=\rho \cos \phi$.

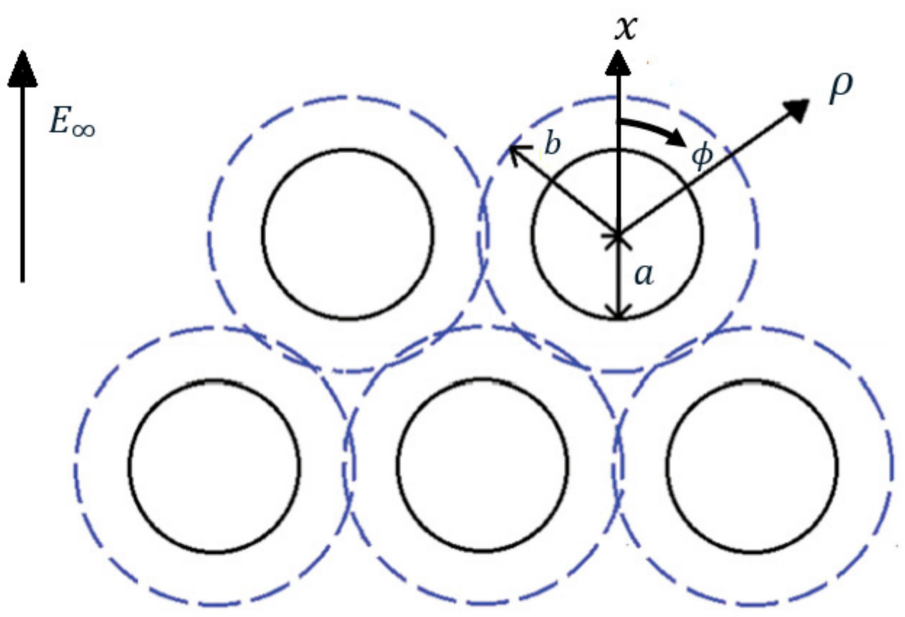

Figure 1. Geometrical sketch of the unit cell model for an array of long parallel circular cylinders.

\subsection{Governing Equations}

The magnitude of the fluid velocity is taken to be small, such that the electric double layer encompassing the cylinder is just distorted slightly from equilibrium. Thus, the electric potential distribution $\psi(\rho, \phi)$, the concentration distribution $n_{m}(\rho, \phi)$ of the ionic species $m$, and the pressure distribution $p(\rho, \phi)$ may be written as in Equation (1):

$$
\psi=\psi^{(\mathrm{eq})}+\delta \psi, \quad n_{m}=n_{m}^{(\mathrm{eq})}+\delta n_{m}, \quad p=p^{(\mathrm{eq})}+\delta p,
$$


where $\psi^{(\mathrm{eq})}(\rho), n_{m}^{(\mathrm{eq})}(\rho)$, and $p^{(\mathrm{eq})}(\rho)$ represent the equilibrium profiles of the electric potential, concentration of the species $m$, and pressure, respectively (with $n_{m}^{(\text {eq) }}$ relating to $\psi^{(\mathrm{eq})}$ by the Boltzmann equation), and $\delta \psi(\rho, \phi), \delta n_{m}(\rho, \phi)$, and $\delta p(\rho, \phi)$ are the small perturbations to equilibrium. Note that $\delta \psi(\rho, \phi)$ contains the electric potentials contributed by both the applied electric field itself and the charge regulation effect at the cylinder's surface induced by the applied electric field.

The perturbed quantities $\delta \psi, \delta n_{m}$, and $\delta p$ along with the fluid velocity distribution $\mathbf{u}(\rho, \phi)$ satisfy the continuity equation $\nabla \cdot \mathbf{u}=0$ and the following linearized electrokinetic equations [10]:

$$
\begin{gathered}
\nabla^{2} \delta \psi=-\frac{e}{\varepsilon k T} \sum_{m=1}^{M} z_{m} n_{m}^{\infty} \exp \left(-\frac{z_{m} e \psi^{(\mathrm{eq})}}{k T}\right)\left(\delta \mu_{m}-z_{m} e \delta \psi\right), \\
\nabla^{2} \delta \mu_{m}=\frac{z_{m} e}{k T}\left[\nabla \psi^{(\mathrm{eq})} \cdot \nabla \delta \mu_{m}-\frac{k T}{D_{m}} \nabla \psi^{(\mathrm{eq})} \cdot \mathbf{u}\right], m=1,2, \cdots, M, \\
\eta \nabla^{2} \mathbf{u}=\nabla \delta p-\varepsilon\left[\nabla^{2} \psi^{(\mathrm{eq})} \nabla \delta \psi+\nabla^{2} \delta \psi \nabla \psi^{(\mathrm{eq})}\right] .
\end{gathered}
$$

In Equations (2) and (3), $\delta \mu_{m}$ is the perturbed ionic electrochemical potential energy defined by

$$
\delta \mu_{m}=z_{m} e \delta \psi+\frac{k T}{n_{m}^{(\mathrm{eq})}} \delta n_{m}
$$

$n_{m}^{\infty}, D_{m}$, and $z_{m}$ are the bulk concentration, diffusion coefficient, and valence, respectively, of the species $m ; \eta$ and $\varepsilon$ are the viscosity and dielectric permittivity, respectively, of the fluid; $e$ is the charge of a proton; $k$ is the Boltzmann constant; $T$ is the absolute temperature. Note that the first parts of Equation (1) and Equation (2) may not be needed to solve for the linear electroosmotic velocity (in terms of the applied electric field strength) of the fluid if the charge regulation effect is absent at the cylinder surface.

\subsection{Boundary Conditions}

The boundary conditions for $\delta \mu_{m}$ and $\mathbf{u}$ at the surface of the cylinder are

$$
\rho=a: \quad \frac{\partial \delta \mu_{m}}{\partial \rho}=0, \quad \mathbf{u}=0,
$$

which are obtained from the assumptions that the shear plane coincides with the cylinder surface and no ions can penetrate into the cylinder. To obtain the boundary condition for the perturbed quantity $\delta \psi$ at the charge-regulating surface, we adopt the linearized regulation model proposed by Carnie and Chan [22] and express the surface charge density $\sigma$ as a linear function of the surface potential $\psi_{\mathrm{S}}$,

$$
\sigma=\sigma^{(\mathrm{eq})}+\left(\frac{\mathrm{d} \sigma}{\mathrm{d} \psi_{\mathrm{S}}}\right)_{\psi_{\mathrm{S}}=\zeta} \delta \psi_{\mathrm{S}}
$$

where the constants $\sigma^{(\mathrm{eq})}$ and $\zeta$ are the values of $\sigma$ and $\psi_{\mathrm{s}}$, respectively, at equilibrium. Note that $\zeta$ does not coincide with the actual interfacial electric potential, which is not assumed to be constant when the external electric field is present. The substitution of Equations (1) and (7) into the Gauss condition at the cylinder surface,

$$
\rho=a: \quad \frac{\partial \psi}{\partial \rho}=-\frac{\sigma}{\varepsilon},
$$

results in

$$
\rho=a: \quad \frac{\partial \delta \psi}{\partial \rho}=-L \delta \psi=0,
$$


where the charge regulation coefficient $L$ is defined and related to measurable quantities by Equation (A6) in Appendix A for the mechanism of charge regulation. The limits $L \rightarrow \infty$ and $L=0$ reduce to the simple cases of constant surface potential and constant surface charge density, respectively.

The boundary conditions at the outer (virtual) surface of the unit cell may be

$$
\begin{gathered}
\rho=b: \quad \frac{\partial \delta \psi}{\partial \rho}=-E_{\infty} \cos \phi, \\
\frac{\partial \delta \mu_{m}}{\partial \rho}=-z_{m} e E_{\infty} \cos \phi, \\
u_{\rho}=-U \cos \phi, \\
\tau_{\rho \phi}=\eta\left[\rho \frac{\partial}{\partial \rho}\left(\frac{u_{\phi}}{\rho}\right)+\frac{1}{\rho} \frac{\partial u_{\rho}}{\partial \phi}\right]=0
\end{gathered}
$$

for the Happel model;

$$
(\nabla \times \mathbf{u})_{z}=\frac{1}{\rho} \frac{\partial}{\partial \rho}\left(\rho u_{\phi}\right)-\frac{1}{\rho} \frac{\partial u_{\rho}}{\partial \phi}=0
$$

for the Kuwabara model, where $u_{\rho}$ and $u_{\phi}$ are the $\rho$ and $\phi$ components, respectively, of $\mathbf{u}$ and $U$ is the superficial electroosmotic velocity of the fluid (may also be taken as the electrophoretic velocity of the circular cylinder in the opposite direction in a quiescent fluid) to be determined. In Equations (10a) and (10b), the local electric field consistent with the imposed electric field $E_{\infty} \mathbf{e}_{x}$ is set according to the Levine-Neale electrostatic model [6].

Alternatively, the electric potential at the outer surface of the cell may be set as the profile resulting in the imposed electric field $E_{\infty} \mathbf{e}_{x}$, known as the Zharkikh-Shilov electrostatic model [7], and the boundary conditions in Equations (10a) and (10b) become

$$
\rho=b: \quad \delta \psi=-E_{\infty} \rho \cos \phi, \quad \delta \mu_{m}=-z_{m} e E_{\infty} \rho \cos \phi .
$$

Note that the electric field strength at infinity $E_{\infty}$ in the previous equation might be replaced more appropriately by the volume-mean electric field strength [12].

\subsection{Equilibrium Electric Potential}

To solve for the electroosmotic velocity in a unit cell, we need the equilibrium electric potential distribution $\psi^{(\mathrm{eq})}$ satisfying the Poisson-Boltzmann equation and the boundary conditions

$$
\begin{gathered}
\rho=a: \quad \psi^{(\mathrm{eq})}=\zeta, \\
\rho=b: \quad \frac{\mathrm{d} \psi^{(\mathrm{eq})}}{\mathrm{d} \rho}=0 .
\end{gathered}
$$

For the case of small equilibrium surface (zeta) potential $\zeta$ of the cylinder, it can be shown that

$$
\psi^{(\mathrm{eq})}=\psi_{\mathrm{eq} 1}(\rho) \bar{\zeta}
$$

to the order of $\bar{\zeta}$, where $\bar{\zeta}=\zeta e / k T$,

$$
\psi_{\mathrm{eq} 1}(\rho)=\frac{k T}{e} \frac{I_{1}(\kappa b) K_{0}(\kappa \rho)+I_{0}(\kappa \rho) K_{1}(\kappa b)}{I_{1}(\kappa b) K_{0}(\kappa a)+I_{0}(\kappa a) K_{1}(\kappa b)},
$$

$\kappa$ is the Debye screening parameter equal to $e\left(\sum_{m=1}^{M} z_{m}^{2} n_{m}^{\infty} / \varepsilon k T\right)^{1 / 2}$, and $I_{n}$ and $K_{n}$ are the modified Bessel functions of order $n$ of the first and second kinds, respectively. 
By substituting Equations (14) and (15) into the Gauss condition, one obtains the following formula relating the zeta potential and surface charge density of the cylinder at equilibrium:

$$
\sigma^{(\mathrm{eq})}=\varepsilon \kappa \zeta \frac{I_{1}(\kappa b) K_{1}(\kappa a)-I_{1}(\kappa a) K_{1}(\kappa b)}{I_{1}(\kappa b) K_{0}(\kappa a)+I_{0}(\kappa a) K_{1}(\kappa b)} .
$$

The zeta potential $\zeta$ of the charge-regulating cylinder can be evaluated in terms of measurable quantities by using Equations (16) and (A3) (with $\delta \mu_{\mathrm{s}}=0$ at equilibrium). For the limiting case of $\varphi=(a / b)^{2}=0$, Equation (16) reduces to the simple relation $\sigma^{(\mathrm{eq})}=\varepsilon \kappa \zeta K_{1}(\kappa a) / K_{0}(\kappa a)$ for an isolated charged cylinder.

\subsection{Perturbed Quantities and Electroosmotic Velocity}

The small perturbed quantities $\delta \psi, \delta \mu_{m}, v_{r}, v_{\theta}$, and $\delta p$ satisfying Equations (2)-(11) and the continuity equation $\nabla \cdot \mathbf{v}=0$ can be solved in terms of $\psi_{\text {eq } 1}$ in Equation (15), and the results to the sufficient (zeroth or first) orders of $\bar{\zeta}$ for determining the electroosmotic velocity in the fibrous medium correct to the order of $\bar{\zeta}$ are

$$
\begin{gathered}
\delta \psi=E_{\infty} F_{\psi}(\rho) \cos \phi, \\
\delta \mu_{m}=E_{\infty}\left[F_{m 0}+\bar{\zeta} F_{m 1}(\rho)\right] \cos \phi, \\
u_{\rho}=E_{\infty} \bar{\zeta} F_{\rho}(\rho) \cos \phi, \\
u_{\phi}=E_{\infty} \bar{\zeta} F_{\phi}(\rho) \sin \phi, \\
\delta p=E_{\infty} \bar{\zeta}\left[\frac{\eta}{a} F_{p}(\rho)+\varepsilon \kappa^{2} F_{\psi}(\rho) \psi_{\text {eq } 1}(\rho)\right] \cos \phi,
\end{gathered}
$$

For these solutions, the functions $F_{m 1}(\rho), F_{\rho}(\rho), F_{\phi}(\rho)$, and $F_{p}(\rho)$ were given in [18] but with $\psi_{\text {eq } 1}(\rho)$ expressed by Equation (15):

$$
\begin{gathered}
F_{m 0}=-z_{m} e \frac{\rho}{\chi}\left(1+\frac{a^{2}}{\rho^{2}}\right), \\
F_{\psi}(\rho)=I_{1}(\kappa \rho)\left\{A_{K}(\rho)-\frac{2 h_{K}+\kappa g_{K}\left[h_{K} A_{I}(a)+h_{I} A_{K}(a)\right]}{\kappa\left[g_{I} h_{K}-h_{I} g_{K}\right]}\right\}-K_{1}(\kappa \rho)\left\{A_{I}(\rho)\right. \\
\left.+\frac{2 h_{I}+\kappa g_{I}\left[h_{K} A_{I}(a)+h_{I} A_{K}(a)\right]}{\kappa\left[g_{I} h_{K}-h_{I} g_{K}\right]}\right\}
\end{gathered}
$$

if Equation (10a) is used,

$$
\begin{gathered}
F_{\psi}(\rho)=I_{1}(\kappa \rho)\left\{A_{K}(\rho)-\frac{b h_{K}-K_{1}(\kappa b)\left[h_{K} A_{I}(a)+h_{I} A_{K}(a)\right]}{I_{1}(\kappa b) h_{K}+h_{I} K_{1}(\kappa b)}\right\}-K_{1}(\kappa \rho)\left\{A_{I}(\rho)\right. \\
\left.+\frac{b h_{I}+I_{1}(\kappa b)\left[h_{K} A_{I}(a)+h_{I} A_{K}(a)\right]}{I_{1}(\kappa b) h_{K}+h_{I} K_{1}(\kappa b)}\right\}
\end{gathered}
$$

if Equation (11) is used, where

$$
\begin{gathered}
A_{I}(\rho)=\frac{1}{\chi} \int_{\rho}^{b}(\kappa \rho)^{2} I_{1}(\kappa \rho)\left[1+\left(\frac{a}{\rho}\right)^{2}\right] \mathrm{d} \rho, \\
A_{K}(\rho)=\frac{1}{\chi} \int_{\rho}^{b}(\kappa \rho)^{2} K_{1}(\kappa \rho)\left[1+\left(\frac{a}{\rho}\right)^{2}\right] \mathrm{d} \rho, \\
g_{I}=I_{0}(\kappa b)+I_{2}(\kappa b), \quad g_{K}=K_{0}(\kappa b)+K_{2}(\kappa b), \\
h_{I}=\kappa a I_{0}(\kappa a)-(1+a L) I_{1}(\kappa a), \quad h_{K}=\kappa a K_{0}(\kappa a)+(1+a L) K_{1}(\kappa a),
\end{gathered}
$$


and the coefficient $\chi$ equals $1-\varphi$ for the Levine-Neale model in Equations (10a) and (10b) and $1+\varphi$ for the Zharkikh-Shilov model in Equation (11). Note that the perturbed electric potential $\delta \psi$ given by Equations (17) and (21) corrected by the charge regulation effect at the cylinder surface induced by the applied electric field does not affect the functions $F_{m 0}(\rho)$, $F_{m 1}(\rho), F_{\rho}(\rho), F_{\phi}(\rho)$, and $F_{\rho}(\rho)$ for the perturbed ionic electrochemical potential energy $\delta \mu_{m}$ and the fluid flow, but it does contribute to the perturbed pressure $\delta p$ via Equation (19c) and influence the force balance on the unit cell. The electric potential distribution along the cylinder surface $\psi_{\mathrm{s}}$ may be determined from Equations (1), (14), (15), (17), and (21) by taking $\rho=a$, and then the surface charge density distribution $\sigma$ is obtained from Equation (A3). length is

The hydrodynamic drag force exerted on the unit cell at its virtual surface per unit

$$
\mathbf{F}_{\mathrm{h}}=2 b \int_{0}^{\pi}\left\{-\delta p e_{\rho}+\eta\left[\nabla \mathbf{u}+(\nabla \mathbf{u})^{\mathrm{T}}\right] \cdot e_{\rho}\right\}_{\rho=b} \mathrm{~d} \phi .
$$

Since the whole cell is electrically neutral, both the electrostatic force and the net (hydrodynamic) force exerted on it must be zero. By applying this constraint to Equation (25) (with the substitution of Equation (19)), we obtain the electroosmotic velocity of the fluid correct to the order of $\bar{\zeta}$ as

$$
\begin{gathered}
U=\frac{\varepsilon E_{\infty}(\kappa a)^{2} \bar{\zeta}}{8 \eta\left(1+\phi^{2}\right) \chi}\left\{\frac{\chi F_{\psi}(b) \psi_{\mathrm{eq} 1}(b)}{a \phi^{1 / 2} \omega}-\int_{a}^{b}\left(1+\frac{a^{2}}{\rho^{2}}\right)\left[1-\frac{\rho^{2}}{a^{2}}\left(1-2 \ln \frac{\rho}{a}\right)\right.\right. \\
\left.\left.+\phi^{2} \frac{\rho^{2}}{a^{2}}\left(1+2 \ln \frac{\rho}{a}-\frac{\rho^{2}}{a^{2}}\right)\right] \frac{\mathrm{d} \psi_{\mathrm{eq} 1}}{\mathrm{~d} \rho} \mathrm{d} \rho\right\}
\end{gathered}
$$

for the Happel hydrodynamic model, and

$$
\begin{gathered}
U=\frac{\varepsilon E_{\infty}(\kappa a)^{2} \bar{\zeta}}{8 \eta \chi}\left\{\frac{3 \chi F_{\psi}(b) \psi_{\mathrm{eq} 1}(b)}{2 a \phi^{1 / 2} \omega \prime}-\int_{a}^{b}\left(1+\frac{a^{2}}{\rho^{2}}\right)\left[1-\frac{\rho^{2}}{a^{2}}\left(1-2 \ln \frac{\rho}{a}\right)\right.\right. \\
\left.\left.-\frac{1}{2} \phi\left(1-2 \frac{\rho^{2}}{a^{2}}+\frac{\rho^{4}}{a^{4}}\right)\right] \frac{\mathrm{d} \psi_{\mathrm{eq} 1}}{\mathrm{~d} \rho} \mathrm{d} \rho\right\}
\end{gathered}
$$

for the Kuwabara hydrodynamic model, where

$$
\omega=\left(1-\varphi^{2}+\ln \varphi+\varphi^{2} \ln \varphi\right)^{-1}, \quad \omega^{\prime}=\left(1-\frac{4}{3} \varphi+\frac{1}{3} \varphi^{2}+\frac{2}{3} \ln \varphi\right)^{-1} .
$$

When Equation (11) for the Zharkikh-Shilov electrostatic model is used, Equation (26) (with $\chi=1+\varphi$ ) for $U$ will not depend on the charge regulation coefficient $L$. Equation (26) can also be used to express the electrophoretic velocity of a suspension of parallel circular cylindrical particles in the direction normal to their axes.

\subsection{Electric Conductivity}

For an array of parallel charge-regulating circular cylinders subject to a transversely imposed electric field, the effective electric conductivity correct in the order of $\bar{\zeta}$ can be obtained as

$$
\begin{gathered}
\Lambda=\Lambda^{\infty}+\frac{e}{\pi b k T} \sum_{m=1}^{M} z_{m} n_{m}^{\infty} D_{m} \int_{0}^{2 \pi}\left\{F_{m 0}-\rho \frac{\partial F_{m 0}}{\partial \rho}\right)+\bar{\zeta}\left[\rho\left(\frac{z_{m} e}{k T} \psi_{\mathrm{eq} 1} \frac{\partial F_{m 0}}{\partial \rho}-\frac{\partial F_{m 1}}{\partial \rho}\right)\right. \\
\left.\left.+F_{m 1}-\frac{z_{m} e}{k T} \psi_{\mathrm{eq} 1}\left(F_{m 0}-z_{m} e F_{\psi}\right)\right]\right\}_{\rho=b} \mathrm{~d} \phi,
\end{gathered}
$$

where $\Lambda^{\infty}=e^{2} \sum_{m=1}^{M} z_{m}^{2} n_{m}^{\infty} D_{m} / k T$ is the electric conductivity of the ionic fluid in the absence of the cylinders. 
By substituting Equations (15), (20), and (21) together with $F_{m 1}(\rho)$ into Equation (28) and making relevant calculations, we can obtain the effective electric conductivity in the fibrous medium as

$$
\Lambda=\Lambda^{\infty}\left(H+H^{2} I \beta \bar{\zeta}\right)
$$

for the Levine-Neale model, and

$$
\Lambda=\Lambda^{\infty}(H+I \beta \bar{\zeta})
$$

for the Zharkikh-Shilov model. In these expressions,

$$
\begin{gathered}
\beta=\frac{\sum_{m=1}^{M} z_{m}^{3} n_{m}^{\infty} D_{m}}{\sum_{m=1}^{M} z_{m}^{2} n_{m}^{\infty} D_{m}}, \\
H=\frac{1-\varphi}{1+\varphi^{\prime}} \\
I=\frac{e}{k T}\left[\frac{\varphi^{1 / 2}}{a} \psi_{\mathrm{eq} 1}(b) F_{\psi}(b)+2 \frac{\varphi}{\chi} \psi_{\mathrm{eq} 1}(b)-\frac{\varphi}{\chi^{2}} \int_{a}^{b}\left(\frac{a^{2}}{\rho^{2}}-\frac{\rho^{2}}{a^{2}}\right) \frac{\mathrm{d} \psi_{\mathrm{eq} 1} \mathrm{~d} \rho}{\mathrm{d} \rho}\right],
\end{gathered}
$$

Note that the dimensionless coefficient $I$ is irrelevant to the hydrodynamic boundary condition at the outer edge of the unit cell since the effect of electroosmosis on $\Lambda$ does not appear to the order of $\bar{\zeta}$. When Equation (11) for the Zharkikh-Shilov electrostatic model is used, the result for the coefficient $I$ [given by Equation (32) with $\chi=1+\varphi$ ] will not depend on the charge regulation coefficient $L$.

\section{Results and Discussion}

\subsection{Charge Regulation Characteristics}

Before calculating the electroosmotic velocity and electric conductivity in the array of charge-regulating circular cylinders from Equations (26), (29)-(32), we present typical results of the dimensionless equilibrium surface charge density $e \sigma^{(\mathrm{eq})} / \varepsilon \kappa k T$, zeta potential $\bar{\zeta}$, and charge regulation parameter $L a$ as functions the bulk concentration $n_{1}^{\infty}$ of the charge-determining ions, equilibrium constants $K_{+}$and $K_{-}$of the surface reactions in Equations (A1) and (A2), respectively, and porosity $1-\varphi$ of the fibrous medium in Figures 2-4 using Equation (16), (A3), and (A6) (independent of the electrokinetic conditions at the outer boundary of the unit cell). The aqueous 1-1 electrolyte solution at $T=298 \mathrm{~K}$ (with dielectric constant 78.54) and the cylinders of radius $a=0.1 \mu \mathrm{m}$ with amphoteric functional group density $N=1 \times 10^{16}$ site $/ \mathrm{m}^{2}$ are chosen. When the bulk concentration of the charge-determining ions is smaller than that of the isoelectric point $\left[n_{1}^{\infty}<\left(K_{+} K_{-}\right)^{1 / 2}\right]$, the values of $\sigma^{(\mathrm{eq})}$ and $\zeta$ are negative as expected; the magnitude of $\bar{\zeta}$ increases monotonically with a decrease in $n_{1}^{\infty}$ keeping the other parameters unchanged, whereas the magnitude of $e \sigma^{(\mathrm{eq})} / \varepsilon \kappa k T$ also increases monotonically with a decrease in $n_{1}^{\infty}$ for the case of $\varphi=0$ but may have a maximal magnitude at a finite value of $n_{1}^{\infty}$ for the case of nonzero $\varphi$. When $n_{1}^{\infty}>\left(K_{+} K_{-}\right)^{1 / 2}$, the values of $e \sigma^{(\mathrm{eq})} / \varepsilon \kappa k T$ and $\bar{\zeta}$ are positive and may not be monotonic functions of $n_{1}^{\infty}$.

The magnitudes of $e \sigma^{(\mathrm{eq})} / \varepsilon \kappa k T$ and $\bar{\zeta}$ decrease as $K_{-} / K_{+}$decreases (implying that the concentration of unionized surface groups increases). The cylinders are less positively charged or more negatively charged as $K_{+} K_{-}$increases (implying that the concentration of negatively charged surface groups increases or that of positively charged surface groups decreases). The magnitude of $e \sigma^{(\mathrm{eq})} / \varepsilon \kappa k T$ decreases but that of $\bar{\zeta}$ increases as $\varphi$ increases. The charge regulation parameter $L a$ is not a monotonic function of $n_{1}^{\infty}, K_{+} K_{-}, K_{-} / K_{+}$, and $\varphi$. In general, the dimensionless quantities $e \sigma^{(\mathrm{eq})} / \varepsilon \kappa k T, \bar{\zeta}$, and $L a$ vary sensitively with changes in $n_{1}^{\infty}, K_{+}$, and $K_{-}$, but their dependencies on $\varphi$ are negligible when $n_{1}^{\infty}$ is greater than about $0.1 \mathrm{mM}$. 


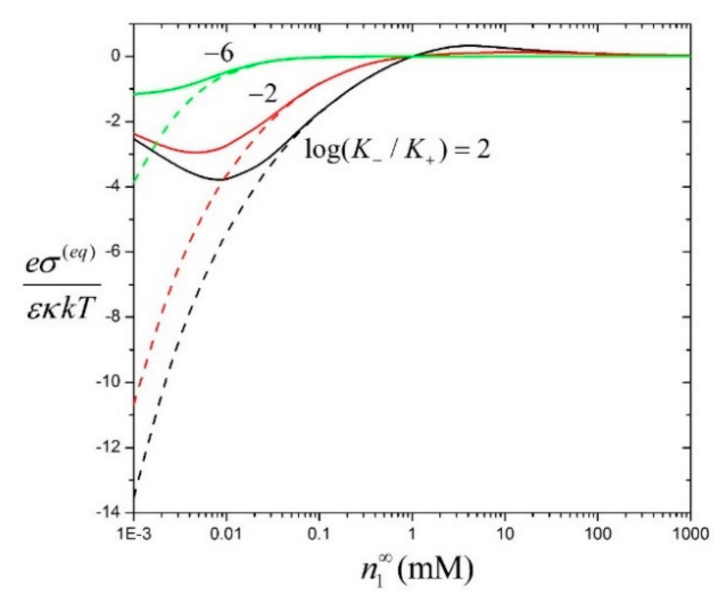

(a)

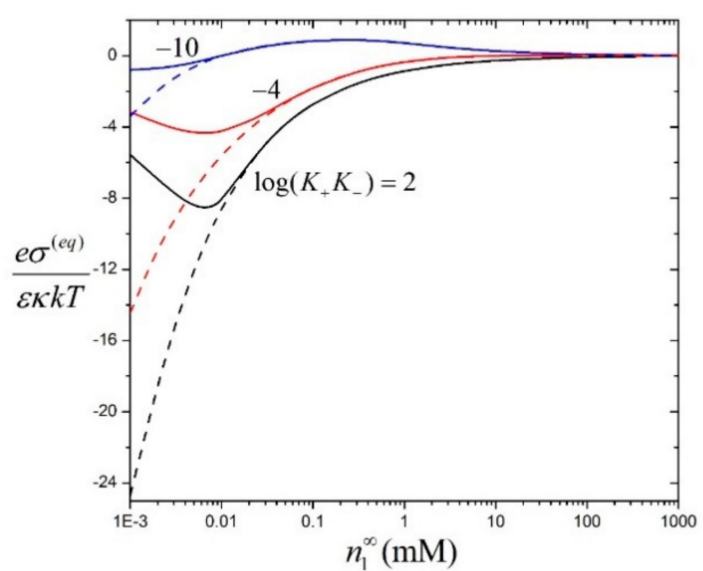

(b)

Figure 2. The dimensionless equilibrium surface charge density e $\sigma^{(\mathrm{eq})} / \varepsilon \kappa k T$ versus the bulk concentration $n_{1}^{\infty}$ of an aqueous 1-1 electrolyte solution in a fibrous medium with $a=100 \mathrm{~nm}$ and $N=1 \times 10^{16} \mathrm{site} / \mathrm{m}^{2}:(\mathbf{a}) K_{+} K_{-}=10^{-6} \mathrm{M}^{2}$; (b) $K_{-} / K_{+}=10^{-2}$. The solid and dashed curves represent the cases $\varphi=0.3$ and $\varphi=0$, respectively.

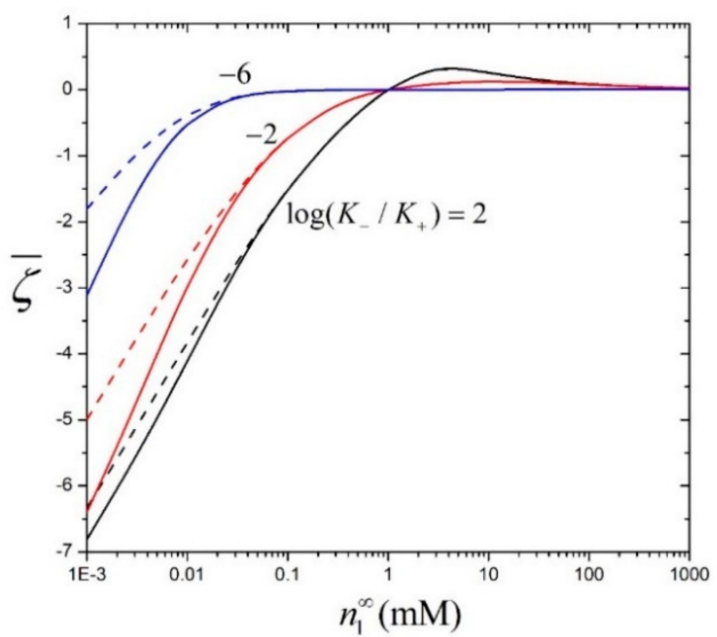

(a)

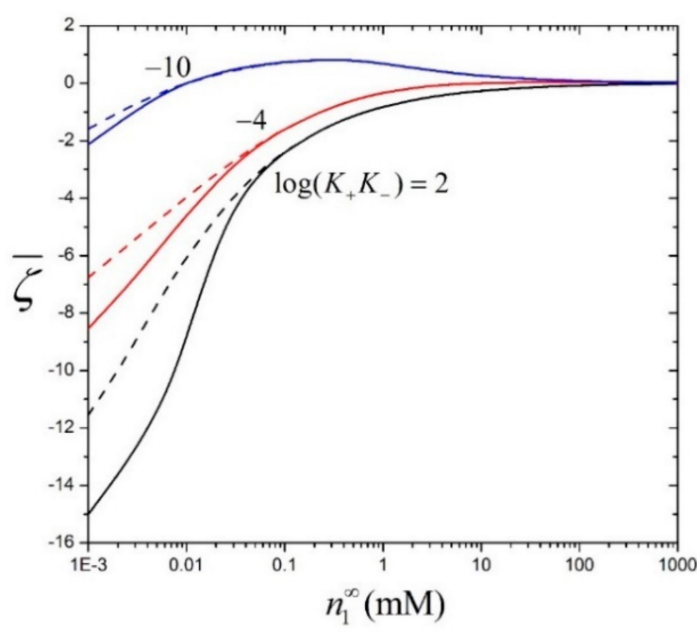

(b)

Figure 3. The dimensionless zeta potential $\bar{\zeta}$ versus the bulk concentration $n_{1}^{\infty}$ of an aqueous 1-1 electrolyte solution in a fibrous medium with $a=100 \mathrm{~nm}$ and $N=1 \times 10^{16} \mathrm{site} / \mathrm{m}^{2}$ : (a) $K_{+} K_{-}=10^{-6} \mathrm{M}^{2} ;(\mathbf{b}) K_{-} / K_{+}=10^{-2}$. The solid and dashed curves represent the cases $\varphi=0.3$ and $\varphi=0$, respectively. 


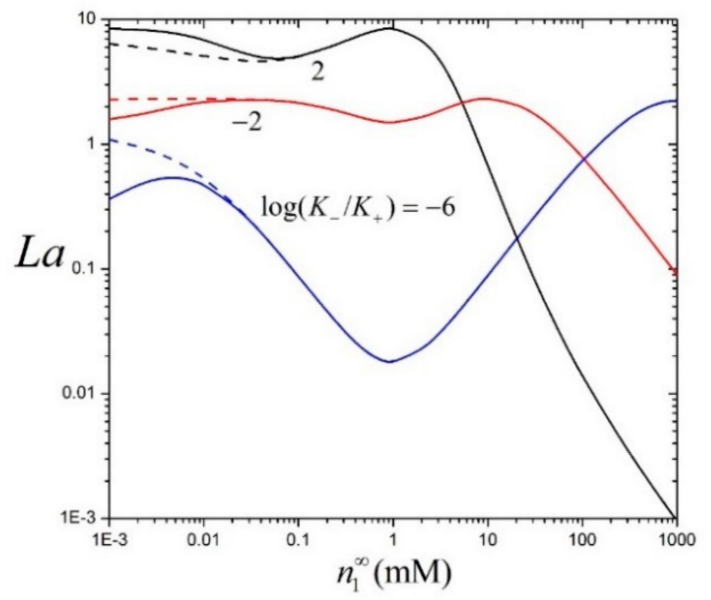

(a)

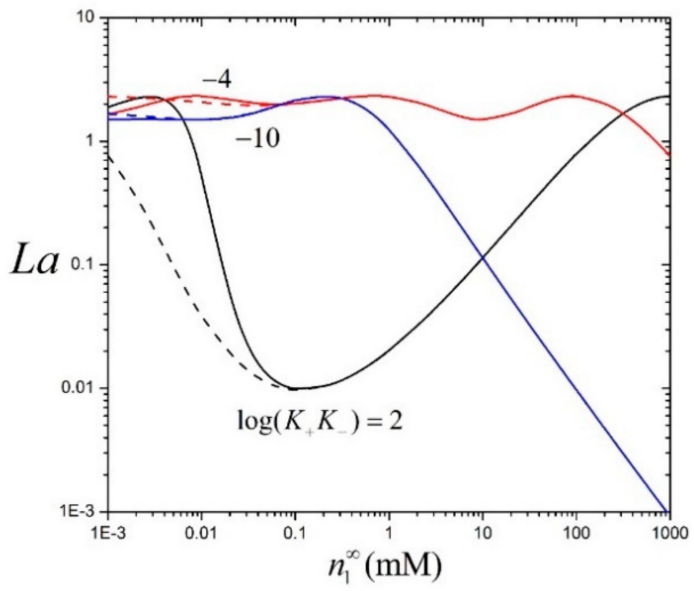

(b)

Figure 4. The dimensionless charge regulation parameter $L a$ versus the bulk concentration $n_{1}^{\infty}$ of an aqueous 1-1 electrolyte solution in a fibrous medium with $a=100 \mathrm{~nm}$ and $N=1 \times 10^{16} \mathrm{site} / \mathrm{m}^{2}$ : (a) $K_{+} K_{-}=10^{-6} \mathrm{M}^{2} ;(\mathbf{b}) K_{-} / K_{+}=10^{-2}$. The solid and dashed curves represent the cases $\varphi=0.3$ and $\varphi=0$, respectively.

\subsection{Electroosmotic Velocity}

Since the electroosmotic velocity of an arbitrary electrolyte solution in the chargeregulating fiber matrix predicted from the Zharkikh-Shilov electrostatic model is independent of the charge regulation coefficient $L$, we plot the normalized electroosmotic velocity $\eta U / \varepsilon \zeta E_{\infty}$ calculated from Equation (26) with $\chi=1-\varphi$ (the Levine-Neale electrostatic model) for various values of the electrokinetic radius $\kappa a$, charge regulation parameter $L a$, and porosity $1-\varphi$ in Figures 5 and 6 for the Happel and Kuwabara hydrodynamic models, respectively. The result is shown up to $\varphi=0.9$, corresponding to the minimum attainable porosity for an array of identical parallel circular cylinders [31]. The velocity $\eta U / \varepsilon \zeta E_{\infty}$ increases moderately and monotonically with a decrease in $L a$ for fixed values of $\varphi$ and $\kappa a$, but becomes independent of $L a$ in the limiting cases of $\varphi=0, \kappa a=0$, and $\kappa a \rightarrow \infty$. For specified values of $L a$ and $\varphi$, this normalized velocity increases monotonically with an increase in $\kappa a$. When $\kappa a=0, U=\varepsilon \zeta E_{\infty} / 2 \eta$ as $\varphi=0$ and $U=0$ for any finite value of $\varphi$.

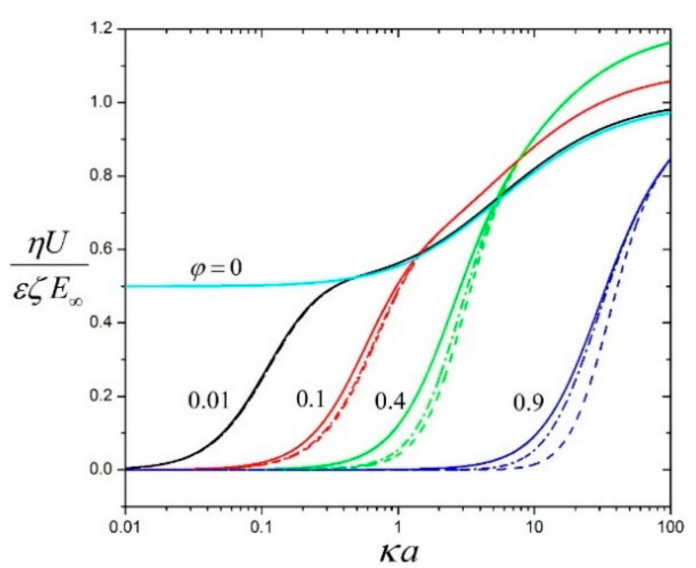

(a)

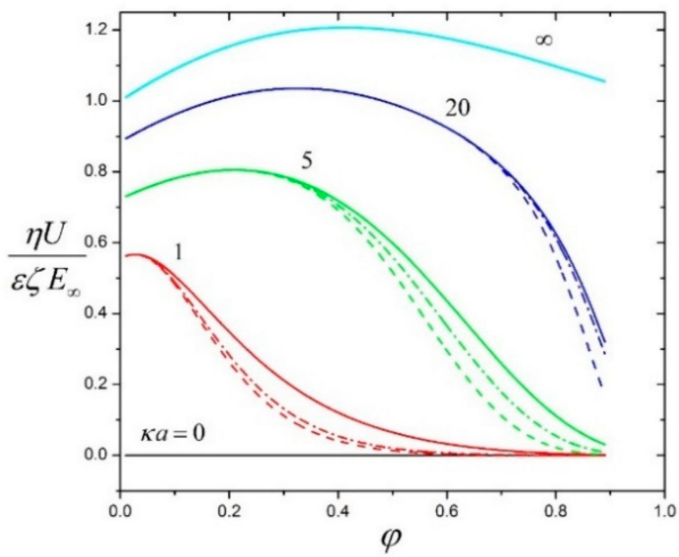

(b)

Figure 5. The normalized electroosmotic velocity $\eta U / \varepsilon \zeta E_{\infty}$ of an electrolyte solution through a fibrous medium calculated from Equation (26a) for the Happel/Levine-Neale cell model versus the parameters $\kappa a$ (a) and $\varphi(\mathbf{b})$. The solid, dot-dash, and dashed curves represent the cases of the charge regulation parameter $L a$ equal to 0,5 , and $\infty$, respectively. 


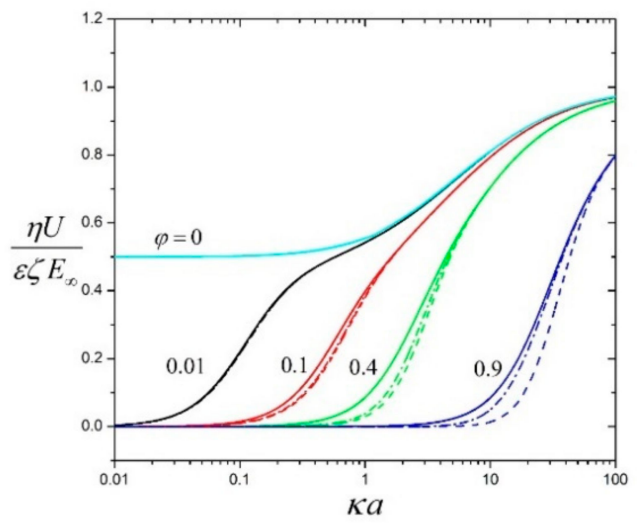

(a)

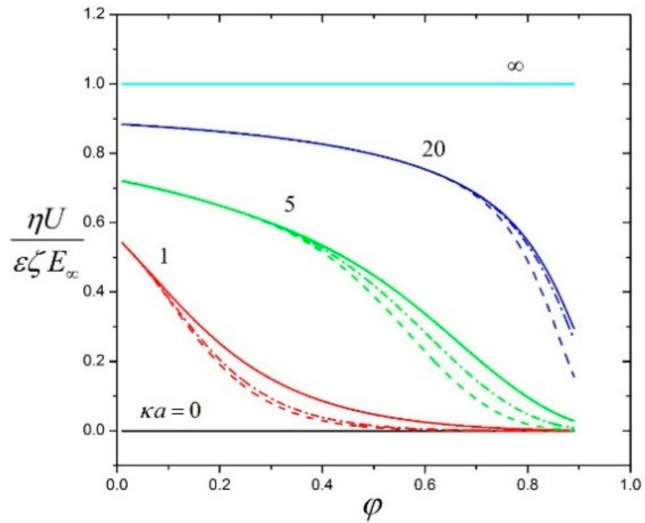

(b)

Figure 6. The normalized electroosmotic velocity $\eta U / \varepsilon \zeta E_{\infty}$ of an electrolyte solution through a fibrous medium calculated from Equation (26b) for the Kuwabara/Levine-Neale cell model versus the parameters $\kappa a(\mathbf{a})$ and $\varphi(\mathbf{b})$. The solid, dot-dash, and dashed curves represent the cases of the charge regulation parameter $L a$ equal to 0,5 , and $\infty$, respectively.

For the Kuwabara model, $\eta U / \varepsilon \zeta E_{\infty}$ decreases monotonically and significantly with an increase in $\varphi$ for given values of $L a$ and $\kappa a$ and always equals unity as $\kappa a \rightarrow \infty$. For the Happel model, however, $\eta U / \varepsilon \zeta E_{\infty}$ is no longer a monotonic function of $\varphi$ but has a maximum (whose value can even be larger than unity) at some moderate value of $\varphi$ for a constant value of $\kappa a$ larger than about 0.7. The location of this maximum shifts to smaller $\varphi$ as $\kappa a$ decreases. For a fixed value of $\kappa a$ smaller than about 0.6 , similarly to the Kuwabara model, $\eta U / \varepsilon \zeta E_{\infty}$ becomes a monotonic decreasing function of $\varphi$. The Happel model always predicts a greater value of $\eta U / \varepsilon \zeta E_{\infty}$ than the Kuwabara model does for any combination of $\kappa a, L a$, and $\varphi$. Our results in the limit $L a=0$ (solid curves in Figures 5 and 6 for the case of constant surface charge density) agree with those obtained previously [18]. Note that, although the normalized electroosmotic velocity $\eta U / \varepsilon \zeta E_{\infty}$ just varies moderately (for the Levine-Neale model) or does not vary at all (for the Zharkikh-Shilov model) with the change in the charge regulation parameter $L a$, the dimensional electroosmotic velocity $U$ still varies substantially with the change in the charge regulation characteristics such as $n_{1}^{\infty}, K_{+}$, and $K_{-}$since the zeta potential $\zeta$ varies strongly with these characteristics as shown in Figure 3.

\subsection{Electric Conductivity}

The dimensionless coefficient $I$ in Equation (29) for the effective electric conductivity in the charge-regulating fibrous medium is independent of the hydrodynamic boundary condition prescribed at the virtual surface of the unit cell and that predicted from the Zharkikh-Shilov electrostatic model [given by Equation (32) with $\chi=1+\varphi$ ] is independent of the charge regulation coefficient $L$. In Figure 7, the coefficient $H^{2} I$ in Equation (29) calculated from Equations (31) and (32) with $\chi=1-\varphi$ (the Levine-Neale electrostatic model) is plotted for various values of the electrokinetic radius $\kappa a$, charge regulation parameter $L a$, and porosity $1-\varphi$. For the case of $L a=0$ (constant surface charge density), $H^{2} I$ is always negative and thus the presence of the surface charge decreases and increases the effective electric conductivity for any porosity of the fiber matrix if $\beta \zeta>0$ and $\beta \zeta<0$, respectively. The coefficient $H^{2} I$ decreases with a decrease in $L a$ for specified values of $\kappa a$ and $\varphi$, but this dependence becomes weak as $\kappa a$ is large or $\varphi$ is small. Interestingly, for the combination of small $\kappa a$, large $\varphi$, and large $L a$, the value of $H^{2} I$ may become positive and thus the presence of the surface charge increases and decreases the effective conductivity if $\beta \zeta>0$ and $\beta \zeta<0$, respectively. 


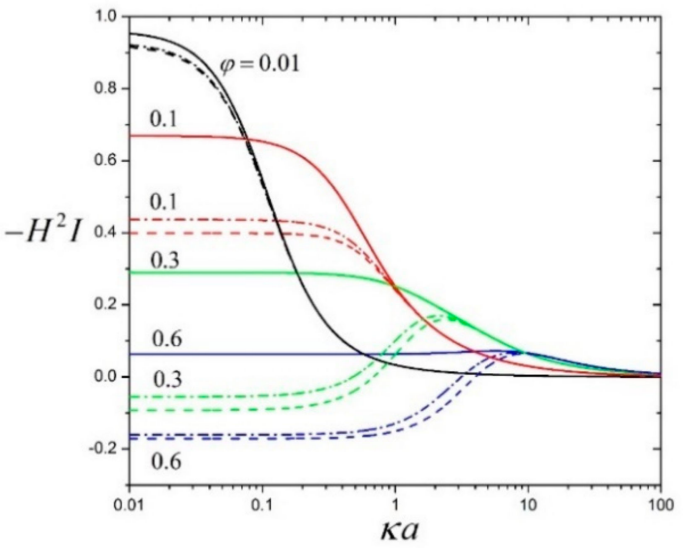

(a)

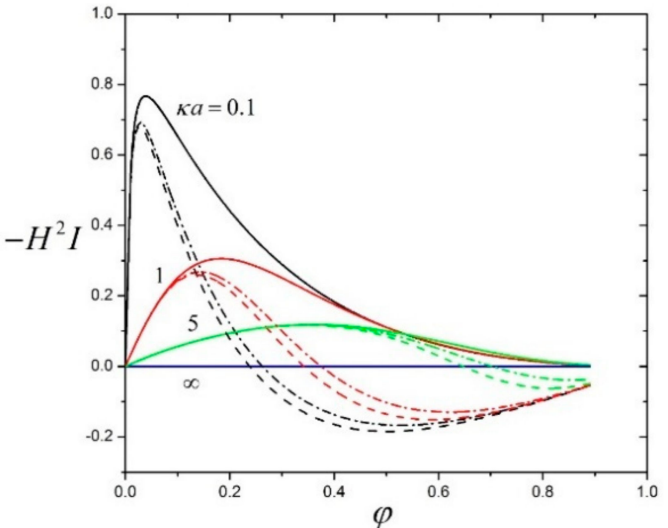

(b)

Figure 7. The dimensionless coefficient $H^{2} I$ in Equation (29a) for the Levine-Neale cell model for the effective electric conductivity in a fibrous medium calculated from Equations (31) and (32) versus the parameters $\kappa a(\mathbf{a})$ and $\varphi$ (b). The solid, dot-dash, and dashed curves represent the cases of the charge regulation parameter La equal to 0,5 , and $\infty$, respectively.

The coefficient $H^{2} I$ has a minimum at a finite value of $\varphi$ and vanishes as $\varphi=0$ for fixed values of $\kappa a$ and $L a$, and a maximum of $H^{2} I$ may also exist at a larger value of $\varphi$ when $L a$ is large. The locations of these extrema shift to smaller $\varphi$ as $\kappa a$ decreases or $L a$ increases. The coefficient $H^{2} I$ increases with an increase in $\kappa a$ if either $\varphi$ or $L a$ is small, is not a monotonic function of $\kappa a$ if both $\varphi$ and $L a$ are large, and vanishes as $\kappa a \rightarrow \infty$ irrespective of $\varphi$ and $L a$. Our results in the limit $L a=0$ (solid curves in Figure 7) agree with those obtained previously [18]. Although $H^{2} I$ may vary moderately (for the Levine-Neale model as $\kappa a$ is large or $\varphi$ is small) or does not vary at all (for the Zharkikh-Shilov model) with the change in the charge regulation parameter $L a$, the effective electric conductivity $\Lambda$ in Equation (26) still varies substantially with the change in the charge regulation characteristics such as $n_{1}^{\infty}, K_{+}$, and $K_{-}$since $\zeta$ varies strongly with these characteristics.

\section{Concluding Remarks}

In this work, the electroosmosis and electric conduction of an arbitrary electrolyte solution in a fiber matrix constructed by a homogeneous array of parallel charge-regulating circular cylinders with an arbitrary electric double layer thickness were analytically investigated. A linearized charge regulation model was employed for the association and dissociation reactions occurring at the ionogenic functional groups on the surfaces of the cylinders. By solving the linearized electrokinetic equations applicable to a unit cell, we have determined the electroosmotic mobility of the fluid and effective electric conductivity in the fibrous medium. Our results show that the charge regulation characteristics such as the equilibrium constants of the association and dissociation reactions occurring at the cylinder surfaces and the bulk concentration of the potential-determining ions, and the porosity of the fiber matrix significantly, affect the surface charge density and potential, electroosmotic mobility, and effective electric conductivity.

Equations (26) and (29) were obtained on the basis of a small electric potential around the charge-regulating cylinder in a unit cell. Similar formulas for the electrophoretic mobility and effective electric conductivity of suspensions of charged spherical particles with a low zeta potential were shown to give good approximations for the situation of moderate zeta potentials (with errors less than $5 \%$ for $|\bar{\zeta}| \leq 2$ in $\mathrm{KCl}$ solutions) [30,32]. Thus, our results might be used tentatively for in cases of reasonably high zeta potentials.

When the Levine-Neale electrokinetic cell model with Equations (10a) and (10b) is used, Equation (26) for the electroosmotic mobility and Equation (32) for the effective electric conductivity of an arbitrary electrolyte solution in the charge-regulating fibrous medium will be functions of the charge regulation parameter $L a$, and these transport prop- 
erties will not depend on $L a$ when the Zharkikh-Shilov electrokinetic cell model (Equation (11)) is used. Since both the electroosmotic flow and the electric conduction are considered within the linear approach in terms of the external electric field, and the electroosmotic mobility and effective electric conductivity are expressed by the only surface parameter $\zeta$, in which the charge regulation characteristics such as $n_{1}^{\infty}, K_{+}$, and $K_{-}$are embedded, this dependency might be the same for any type of adsorption equilibrium. Accordingly, the predictions containing implicit dependencies on the charge regulation parameter $\mathrm{La}$ by the Levine-Neale model seem to be incorrect. This outcome probably comes from the insufficiency of this model that the tangential (angular) component of the external electric field at the virtual boundary $(\rho=b)$ of the unit cell is not specified. Indeed, in a cell-model analysis of the electrophoresis in a suspension of spherical particles, the Zharkikh-Shilov model predicts the electrophoretic mobility consistently with the ensemble-averaged result (obtained by using the concept of statistical mechanics), whereas the Levine-Neale model does not [33].

Author Contributions: Conceptualization, H.J.K.; methodology, H.J.K. and W.L.C.; investigation, H.J.K. and W.L.C.; writing—original draft preparation, H.J.K.; writing—review and editing, H.J.K.; supervision, H.J.K.; funding acquisition, H.J.K. All authors have read and agreed to the published version of the manuscript.

Funding: This research was funded by the Ministry of Science and Technology, Taiwan (China), grant number MOST 106-2221-E-002-167-MY3.

Conflicts of Interest: The authors declare no conflict of interest. The funders had no role in the design of the study; in the collection, analyses, or interpretation of data; in the writing of the manuscript, or in the decision to publish the results.

\section{Appendix A}

A general model for the charge-regulating surface in contact with an electrolyte solution to result in fixed charge includes the following association and dissociation reactions occurring at the amphoteric functional groups $\mathrm{AB}$ distributed uniformly at the surface:

$$
\begin{gathered}
\mathrm{AB}_{2}^{\mathrm{Z}}{ }^{\circ} \mathrm{AB}+\mathrm{B}^{\mathrm{Z}}, \\
\mathrm{AB} \Leftrightarrow \mathrm{A}^{\mathrm{Z}}+\mathrm{B}^{\mathrm{Z}},
\end{gathered}
$$

where $\mathrm{B}^{\mathrm{Z}+}$ denotes the potential- or charge-determining ion with the valence $\mathrm{Z}$ (e.g., $\mathrm{H}^{+}$ with $Z=1$ ). By utilizing the Boltzmann distribution for the equilibrium concentration of $\mathrm{B}^{\mathrm{Z}}$, one can express the surface charge density as

$$
\sigma=Z e N \frac{\left[\mathrm{AB}_{2}^{Z+}\right]-\left[\mathrm{A}^{\mathrm{Z}-}\right]}{[\mathrm{AB}]+\left[\mathrm{AB}_{2}^{Z+}\right]+\left[\mathrm{A}^{\mathrm{Z}}\right]}=\operatorname{ZeN} \frac{\Delta \sinh \left\{\left[\mathrm{Ze}\left(\psi_{\mathrm{N}}-\psi_{\mathrm{s}}\right)+\delta \mu_{\mathrm{s}}\right] / k T\right\}}{1+\Delta \cosh \left\{\left[\mathrm{Ze}\left(\psi_{\mathrm{N}}-\psi_{\mathrm{s}}\right)+\delta \mu_{\mathrm{s}}\right] / k T\right\}},
$$

where

$$
\begin{gathered}
\Delta=2\left(\frac{K_{-}}{K^{+}}\right)^{1 / 2}, \\
\psi_{\mathrm{N}}=\frac{k T}{Z e} \ln \frac{n_{1}^{\infty}}{\left(K_{+} K_{-}\right)^{1 / 2}},
\end{gathered}
$$

$N$ is the number of surface groups AB per unit area, $\psi_{\mathrm{s}}$ is the surface potential, $\delta \mu_{\mathrm{s}}$ is the perturbed electrochemical potential energy (defined by Equation (5)) of $\mathrm{B}^{\mathrm{Z}+}$ at the surface from equilibrium, $n_{1}^{\infty}$ is the bulk concentration of $\mathrm{B}^{Z+}, K_{+}$and $K_{-}$are the equilibrium constants of the surface reactions (A1) and (A2), respectively, and $\psi_{\mathrm{N}}$ is the Nernst potential, 
which vanishes at the point of zero charge (with $\left.n_{1}^{\infty}=\left(K_{+} K_{-}\right)^{1 / 2}\right)$. Equation (A3) leads to the charge regulation coefficient:

$$
L=-\frac{1}{\varepsilon}\left(\frac{\mathrm{d} \sigma}{\mathrm{d} \psi_{\mathrm{s}}}\right)_{\psi_{\mathrm{s}}=\zeta}=\frac{\mathrm{Z}^{2} e^{2} N \Delta\left\{\Delta+\cosh \left[Z e\left(\psi_{\mathrm{N}}(\mathrm{eq})-\zeta\right) / k T\right]\right\}}{\varepsilon k T\left\{1+\Delta \cosh \left[Z e\left(\psi_{\mathrm{N}}(\mathrm{eq})-\zeta\right) / k T\right]\right\}^{2}},
$$

whose reciprocal is a length characterizing the charge regulation at the surface, and $\zeta$ is the value of $\psi_{\mathrm{s}}$ at equilibrium.

\section{References}

1. Rice, C.L.; Whitehead, R. Electrokinetic Flow in a Narrow Cylindrical Capillary. J. Phys. Chem. 1965, 69, 4017-4024. [CrossRef]

2. Keh, H.J.; Liu, Y.C. Electrokinetic Flow in a Circular Capillary with a Surface Charge Layer. J. Colloid Interface Sci. 1995, 172, 222-229. [CrossRef]

3. Keh, H.J.; Tseng, H.C. Transient Electrokinetic Flow in Fine Capillaries. J. Colloid Interface Sci. 2001, 242, 450-459. [CrossRef]

4. Chang, C.C.; Wang, C.-Y. Electro-osmotic flow in a sector microchannel. Phys. Fluids 2009, 21, 42002. [CrossRef]

5. Luo, R.H.; Keh, H.J. Electrokinetic flow and electric conduction of salt-free solutions in a capillary. Electrophor. 2020, 41, 1503-1508. [CrossRef] [PubMed]

6. Levine, S.; Neale, G.; Epstein, N. The prediction of electrokinetic phenomena within multiparticle systems. J. Colloid Interface Sci. 1976, 57, 424-437. [CrossRef]

7. Zharkikh, N.I.; Shilov, V.N. Theory of collective electrophoresis of spherical particles in the Henry approximation. Colloid J. USSR 1982, 43, 865-870.

8. Kozak, M.W.; Davis, E. Electrokinetics of concentrated suspensions and porous media. J. Colloid Interface Sci. 1989, 127, 497-510. [CrossRef]

9. Ohshima, H. Electrical Conductivity of a Concentrated Suspension of Spherical Colloidal Particles. J. Colloid Interface Sci. 1999, 212, 443-448. [CrossRef]

10. Ding, J.M.; Keh, H.J. The Electrophoretic Mobility and Electric Conductivity of a Concentrated Suspension of Colloidal Spheres with Arbitrary Double-Layer Thickness. J. Colloid Interface Sci. 2001, 236, 180-193. [CrossRef]

11. Carrique, F.; Cuquejo, J.; Arroyo, F.J.; Jimenez, M.L.; Delgado, A.V. Influence of cell-model boundary conditions on the conductivity and electrophoretic mobility of concentrated suspensions. Adv. Colloid Interface Sci. 2005, 118, 43-50. [CrossRef]

12. Zholkovskij, E.K.; Masliyah, J.H.; Shilov, V.N.; Bhattacharjee, S. Electrokinetic Phenomena in concentrated disperse systems: General problem formulation and Spherical Cell Approach. Adv. Colloid Interface Sci. 2007, 134-135, 279-321. [CrossRef]

13. Liu, H.C.; Keh, H.J. Electrophoresis and electric conduction in a suspension of charged soft particles. Colloid Polym. Sci. 2016, 294, 1129-1141. [CrossRef]

14. Lin, W.C.; Keh, H.J. Diffusiophoresis in Suspensions of Charged Soft Particles. Colloids Interfaces 2020, 4, 30. [CrossRef]

15. Kozak, M.W.; Davis, E. Electrokinetic phenomena in fibrous porous media. J. Colloid Interface Sci. 1986, 112, 403-411. [CrossRef]

16. Ohshima, H. Electroosmotic Velocity in Fibrous Porous Media. J. Colloid Interface Sci. 1999, 210, 397-399. [CrossRef] [PubMed]

17. Hsu, W.T.; Keh, H.J. Electric conductivity in a fibrous porous medium with thin but polarized double layers. Colloid Polym. Sci. 2003, 282, 985-992. [CrossRef]

18. Keh, H.J.; Wu, Y.Y. Electroosmotic Velocity and Electric Conductivity in a Fibrous Porous Medium in the Transverse Direction. J. Phys. Chem. B 2011, 115, 9168-9178. [CrossRef]

19. Chiang, C.C.; Keh, H.J. Transient electroosmosis in the transverse direction of a fibrous porous medium. Colloids Surfaces A: Physicochem. Eng. Asp. 2015, 481, 577-582. [CrossRef]

20. Su, Y.W.; Keh, H.J. Electrokinetic Flow of Salt-Free Solutions in a Fibrous Porous Medium. J. Phys. Chem. B 2019, 123, 9724-9730. [CrossRef] [PubMed]

21. Ninham, B.W.; Parsegian, V. Electrostatic potential between surfaces bearing ionizable groups in ionic equilibrium with physiologic saline solution. J. Theor. Biol. 1971, 31, 405-428. [CrossRef]

22. Carnie, S.L.; Chan, D.Y. Interaction Free Energy between Plates with Charge Regulation: A Linearized Model. J. Colloid Interface Sci. 1993, 161, 260-264. [CrossRef]

23. Ding, J.M.; Keh, H.-J. Electrophoretic Mobility and Electric Conductivity in Dilute Suspensions of Charge-Regulating Composite Spheres. Langmuir 2003, 19, 7226-7239. [CrossRef]

24. Hill, R.J. On the electrophoretic mobility of succinoglycan modelled as a spherical polyelectrolyte: From Hermans-Fujita theory to charge regulation in multi-component electrolytes. J. Colloid Interface Sci. 2016, 482, 131-134. [CrossRef]

25. Philipse, A.; Tuinier, R.; Kuipers, B.; Vrij, A.; Vis, M. On the Repulsive Interaction Between Strongly Overlapping Double Layers of Charge-regulated Surfaces. Colloid Interface Sci. Commun. 2017, 21, 10-14. [CrossRef]

26. Lin, C.Y.; Keh, H.J. Sedimentation Velocity and Potential in Dilute Suspensions of Charge-Regulating Porous Spheres. J. Phys. Chem. B 2019, 123, 3002-3009. [CrossRef]

27. Bharti; Gopmandal, P.P.; Sinha, R.K.; Ohshima, H. Electrophoresis of pH-regulated zwitterionic soft particle: A semi-analytical study. Colloid Polym. Sci. 2020, 298, 79-89. [CrossRef] 
28. Keh, H.J.; Ding, J.M. Electrokinetic flow in a capillary with a charge-regulating surface polymer layer. J. Colloid Interface Sci. 2003, 263, 645-660. [CrossRef]

29. Sadeghi, M.; Saidi, M.H.; Moosavi, A.; Sadeghi, A. Geometry effect on electrokinetic flow and ionic conductance in pH-regulated nanochannels. Phys. Fluids 2017, 29, 122006. [CrossRef]

30. Keh, H.-J.; Ding, J.M. Electrophoretic Mobility and Electric Conductivity of Suspensions of Charge-Regulating Colloidal Spheres. Langmuir 2002, 18, 4572-4583. [CrossRef]

31. Berryman, J.G. Random close packing of hard spheres and disks. Phys. Rev. A 1983, 27, 1053-1061. [CrossRef]

32. O'Brien, R. The electrical conductivity of a dilute suspension of charged particles. J. Colloid Interface Sci. 1981, 81, 234-248. [CrossRef]

33. Wei, Y.K.; Keh, H.-J. Diffusiophoresis and Electrophoresis in Concentrated Suspensions of Charged Colloidal Spheres. Langmuir 2001, 17, 1437-1447. [CrossRef] 